\title{
Drop on demand in a microfluidic chip
}

\author{
Jie Xu and Daniel Attinger \\ Laboratory for Microscale Transport Phenomena, Department of Mechanical Engineering, \\ Columbia University, New York, NY 10027, USA
}

Received 9 November 2007, in final form 22 April 2008

Published 19 May 2008

Online at stacks.iop.org/JMM/18/065020

\begin{abstract}
In this work, we introduce the novel technique of in-chip drop on demand, which consists in dispensing picoliter to nanoliter drops on demand directly in the liquid-filled channels of a polymer microfluidic chip, at frequencies up to $2.5 \mathrm{kHz}$ and with precise volume control. The technique involves a PDMS chip with one or several microliter-size chambers driven by piezoelectric actuators. Individual aqueous microdrops are dispensed from the chamber to a main transport channel filled with an immiscible fluid, in a process analogous to atmospheric drop on demand dispensing. In this paper, the drop formation process is characterized with respect to critical dispense parameters such as the shape and duration of the driving pulse, and the size of both the fluid chamber and the nozzle. Several features of the in-chip drop on demand technique with direct relevance to lab-on-a-chip applications are presented and discussed, such as the precise control of the dispensed volume, the ability to merge drops of different reagents and the ability to move a drop from the shooting area of one nozzle to another for multistep reactions. The possibility to drive the microfluidic chip with inexpensive audio electronics instead of research-grade equipment is also examined and verified. Finally, we show that the same piezoelectric technique can be used to generate a single gas bubble on demand in a microfluidic chip.
\end{abstract}

This article features online multimedia enhancements

(Some figures in this article are in colour only in the electronic version)

\section{Introduction}

The concept of lab on a chip, where a tiny fluid microprocessor performs complex analysis and synthesis tasks relevant to chemistry or biology, has been a subject of academic interest since the early 1990s [1, 2], and there are encouraging signs that industrial lab-on-a-chip applications are growing, exemplified by microfluidic devices for DNA sequencing [3]. The operations needed for biological or chemical analysis are reagent dosing, transport, mixing, splitting, flushing, filtering, analysis, detection and monitoring [4], each operation requiring a precise control in space and time [5]. The microfluidic components needed to perform these operations are buffers, channels, valves, mixers, microheaters and sensors [4-6]. Several achievements, mostly in academia, have demonstrated that shrinking a chemical or biological laboratory into a microchip could have significant benefits such as increased sensitivity, fast response time, low reagent and sample consumptions, as reviewed in [2, 6-8]. The ability to dispense and control small liquid volumes in the microchannels is critical for the lab-on-a-chip technology, and several techniques address, at least partially, this issue. For instance, electrokinetic pinching [9] or the volumetric change induced by a piezoelectric actuator [10] has been used to inject individual liquid plugs in a miscible liquid, followed by successful electrochemical analysis [9]. Also, the segmented flow technique, defined as the transport of two or more immiscible phases in the form of successive plugs in a microchannel, has been developed: it uses syringe pumps feeding two branches of a T-connection [5, 11, 12] or two concentric channels [13, 14], as reviewed in [5]. In the Tconnection process, drops of the so-called dispersed phase are produced in the other liquid phase, the continuous phase, as a result of shear forces and interfacial tension at the T-connection $[15,16]$. The process using two concentric tubes, called flow focusing, initiated by Ganan-Calveo [17], injects the disperse phase from the smaller tube into the continuous phase that flows out of the larger tube, and breakup occurs in an analogous manner as the breakup of an atmospheric jet in atmosphere, which is due to the Plateau-Rayleigh-Savart instability [18-20]. The segmented flow technique decreases mixing times by several orders of magnitude $[5,15]$, while ensuring 

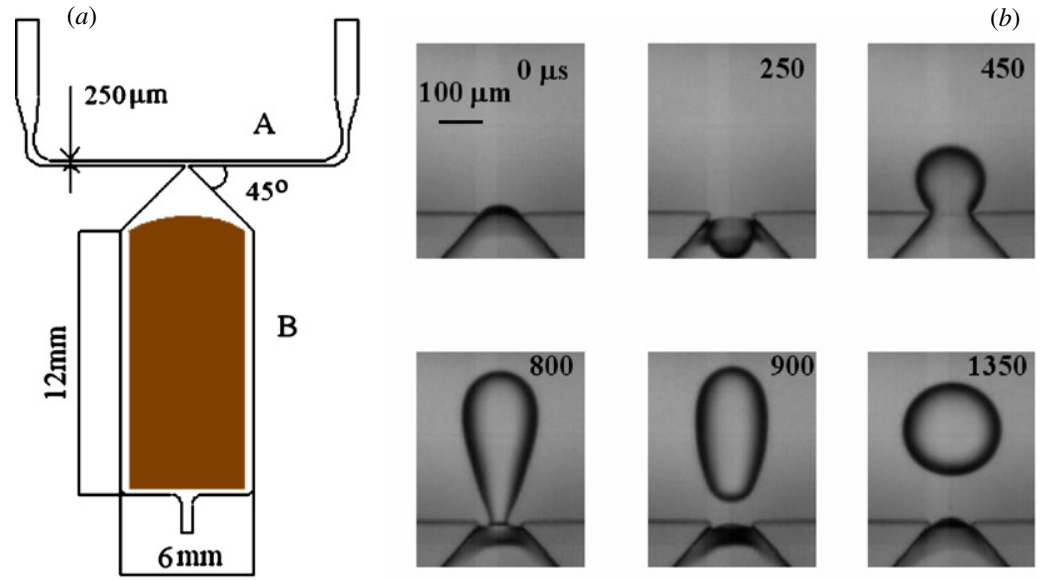

(b)
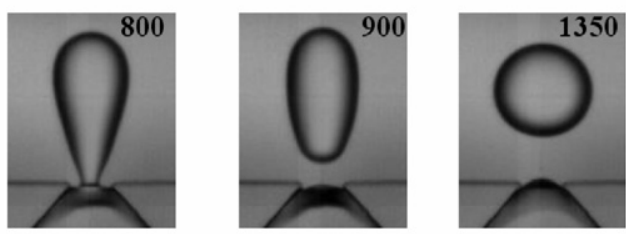

Figure 1. (a) Geometry of a microfluidic chip for in-chip drop on demand dispensing. The bottom chamber is filled with an aqueous reagent. A piezoelectric bimorph actuator glued to the chamber allows the release of an aqueous drop on demand in the horizontal channel filled with an immiscible fluid. (b) Stages depicting the formation of a $1 \mathrm{nl}$ drop from a $50 \mu \mathrm{m}$ nozzle, also shown in the associated movie.

M. An AVI movie of this figure is available from stacks.iop.org/JMM/18/065020

an extremely low diffusion between the two immiscible phases $[5,21]$. These two unique features are extremely attractive for studying and controlling the timing of chemical reactions [21]. Other tasks successfully demonstrated with the segmented flow technique are the splitting of one drop into two halfsize drops using a T-connection [22] and the electrical sorting of drops in the microchannels [23]. A couple of microfluidic systems have also been produced to dose an individual quantity of liquid in the nl- $\mu$ l range [24-31], to be used outside the chip, in a manner analogous to ink-jet printing. Recently, high-performance syringe pumps (microinjectors) [32,33] or a high-voltage pulse have been used to induce the formation a single drop of liquid at a microfluidic T-junction [34, 35], in a dripping-like process. While controlling the drop volume $[32,34]$ and generation timing [35] precisely was difficult, the techniques could be used to encapsulate single cells [32]. In parallel to techniques manipulating droplets in chips, digital microfluidic, also called electrowetting on dielectric, has been developed, which now successfully generates, merges, transports, splits and heats drops with typical volumes of 50 $\mathrm{nl}$ and above, at the surface of a microfluidic chip [4].

The segmented flow and digital microfluidic techniques have the advantage over electrokinetic pinching that the diffusion between the minute amount of liquid dispensed and the carrying fluid is minimal. Both methods are suited for performing complex, multistep analysis or synthesis [4, 21]. Some drawbacks of digital microfluidics in its current state are presented by Fair in [4], such as the need to use conductive liquids, the relatively large volume of the drops (50 $\mathrm{nl}$ and above) and the inability to perform electrokinetic separation useful for chemical characterization. The drawbacks of segmented flow techniques are that a setup might need a relatively long accommodation time before generating the train of particles (drops or bubbles) in a stable manner [36], the difficulty to reliably generate a single particle rather than a train of particles [32-35] and the fact that segmented flow techniques are not as flexible as digital microfluidics for processing multistep reactions [37]. There is therefore a need for a technique that can generate a single particle, on demand, in an immiscible fluid. This need is acknowledged by George Whitesides, who fathered soft microfluidics: 'There is a particular bit of the puzzle that needs to be added, which will not be hard to do but it has not been done yet-that is, bubble on demand [38]'. In this paper, we present a technique to dispense a single drop or bubble on demand in a microfluidic chip. Advantages of this drop and bubble on demand technique over continuous segmented flow techniques can be suggested by looking at the history of atmospheric ink-jet printing [39] and other drop on demand dispense techniques [40-43], where continuous ink-jet printers have been outdated in the 1970s by the invention of drop on demand printers, which add flexibility and eliminate the need for sorting undesired drops.

In section 2, we describe the novel technique to dispense minute, individual amounts of one fluid into another immiscible fluid by ways of piezoelectric actuation, a technique tentatively called in-chip drop on demand. In section 3 , we characterize the dispensing technique. Section 4 describes five features of this technique with direct relevance to the lab-on-a-chip community.

\section{Design, fabrication and setup description}

The typical design of a microfluidic chip used in our study is shown in figure $1(a)$ : it involves one or several $\mu$ l-volume reagent chambers such as B connected via a $25-100 \mu \mathrm{m}$ nozzle to a main channel A. Using syringes, the main channel is filled with a fluid such as hexadecane while each chamber can be filled with a different aqueous reagent. Since the water-hexadecane system is immiscible, a stable meniscus forms at the nozzle opening. The height of the channels is in the $50-100 \mu \mathrm{m}$ range. The chip is sealed with a thin membrane. A piezoelectric actuator is placed on top of each chamber, to modify the chamber volume and release an aqueous drop, on demand, in the main channel. This 
Table 1. Physical properties and typical values.

\begin{tabular}{lll}
\hline Symbol & Physical property & Typical value \\
\hline$\gamma$ & Surface tension at the & $52.5 \mathrm{~mJ} \mathrm{~m}^{-2}[48]$ \\
& water-hexadecane interface & \\
$d_{31}$ & Piezoelectric strain coefficient & $190 \times 10^{-12} \mathrm{~Pa}$ \\
$Y$ & Piezoelectric elastic modulus & $6.2 \times 10^{10} \mathrm{~Pa}$ \\
$\rho$ & PZT density & $7750 \mathrm{~kg} \mathrm{~m}^{-3}$ \\
$E$ & Electric field across actuator & $40-400 \mathrm{kV} \mathrm{m}^{-1}$ \\
$L, B, T$ & Actuator length, width, thickness & $12-20,3-4,0.5 \mathrm{~mm}$ \\
\hline
\end{tabular}

drop generation process is shown in figure $1(b)$ and the associated movie. Once in the main channel, the drop can be transported using viscous drag toward the shooting area of another nozzle, where a drop of another reagent can be dispensed and mixed to the initial drop. This way, a sequence of reactions can be performed by bringing the original drop in front of several chambers shooting prescribed reagents. The internal flow associated with the motion of drops in a channel [10] enhances mixing and diffusion. The needed reagent volume (or dead volume) is relatively small, for instance a chamber of $20 \mathrm{~mm} \times 5 \mathrm{~mm} \times 50 \mu \mathrm{m}$ fed by a tube of $300 \mu \mathrm{m}$ diameter and length $L=1 \mathrm{~cm}$ represents a dead volume of $8 \mu \mathrm{l}$, which corresponds to enough ammunition to shoot $1000080 \mathrm{pl}$ drops.

The microfluidic chips are fabricated in the clean room of Columbia University using soft lithography [44]. First, a $10 \mu \mathrm{m}$ thin base layer of SU-8 2007 resin (MicroChem) is spun and cured on a silicon wafer. On top of that layer, a 50-100 $\mu \mathrm{m}$ layer of SU-8 2050 is cured with patterns transferred from a mask (CAD/Art Services Inc.). This base layer method presented in Carlier et al [45] improves adhesion of $S U-8$ to the wafer. The chip is then manufactured from the master using PDMS Sylgard 184 Kit (Dow Corning). The channels are sealed by a thin $180 \mu \mathrm{m}$ membrane made from spin-coated PDMS. The piezoelectric actuators are commercially available bimorph actuators made of two PZT layers bonded on a thin brass layer, with lengths and widths slightly smaller than the chamber dimensions as shown in figure $1(a)$ and as given in table 1. One actuator is then taped on top of each chamber, using a $90 \mu \mathrm{m}$ layer of double-sided tape.

The experimental setup shown in figure 2 involves three subsystems: the microfluidic system, the actuation system and the sensing system. The microfluidic system involves the microfluidic chip described above (letter ' $a$ ' in figure 2). Syringes fill the main channel with hexadecane and control the subsequent injection of aqueous plugs in the dispensing chamber. The actuation system uses a $20 \mathrm{MHz}$ function generator (letter ' $d$ ', Agilent, 33120A) coupled to a $1 \mathrm{MHz}$ $17 \mathrm{~W}$ amplifier (letter ' $e$ ', Krohn-Hite, 7600M), which generates high-voltage driving pulses for the actuators glued on the microfluidic chip $(a)$. The sensing system is a high-speed high-resolution imaging system involving an Olympus IX-71 microscope $(c)$ and a high-speed camera (letter ' $b$ ', Redlake MotionXtra HG-100K, up to 100000 frames per second). A common point of microfluidic devices involving electrokinetic pinching, segmented flow and digital microfluidics is that

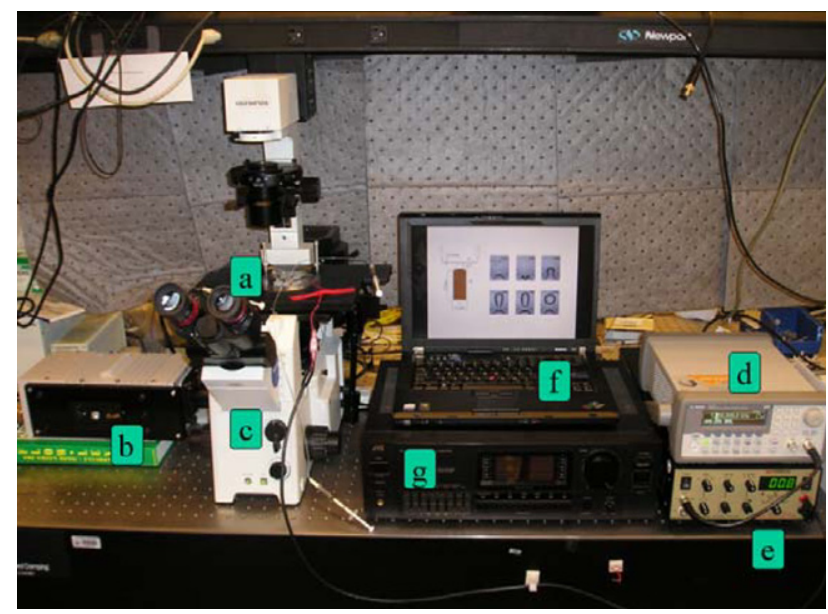

Figure 2. Description of the experimental setup. The microfluidic chip $(a)$ is actuated either by a function generator $(d)$ and a high-voltage amplifier $(e)$ or by the soundcard of computer $(f)$ and an audio amplifier $(g)$. Sensing is performed by the high-speed camera $(b)$ and the inverted microscope $(c)$. Note the scale paradox between the tiny microfluidic chip and the bulky actuation and sensing components, typical of current microfluidic systems.

these techniques require actuation and detection devices that are orders of magnitude larger and more expensive than the chip itself, such as high-voltage power supplies, syringe pumps, drive electronics or microscopes, as seen in figure 2 . There is obviously a real need for reducing the size and cost of microfluidic actuators and sensors, exemplified by the development of simple, portable microfluidic devices in the Whitesides group [46] and by the CMOS-based sensing chips of the Shepard group [47] at Columbia University.

\section{Analysis and characterization}

While our system is a first working prototype, thus far from optimal, this section provides first-order analysis and characterization data that will help building next generations of devices. Section 3.1 compares the energy needed and supplied for producing the drop, section 3.2 describes the motion of the excited actuators and section 3.3 studies the relation between the pulse shape and duration and the drop generation.

\subsection{Energy considerations}

While a full study of how energy is transmitted from the moving actuator to the resulting drop via the soft rubber and the fluid in the three-dimensional, flexible chamber is out of the scope of this paper, we are comparing in this section the energy needed to form a drop with the deformation energy of the actuator. Indeed, the process of generating a microdrop of water in oil (figure $1(b)$ ) involves the sudden excitation of a piezoelectric actuator, which compresses or expands the reagent chamber. This excites the water-oil interface, which eventually breaks up and forms a drop. The minimum energy $U_{d}$ needed to form a drop such as that in figure $1(b)$ can be described as $U_{d}=U_{s}$, where $U_{s}=\pi d^{2} \gamma$, with properties 


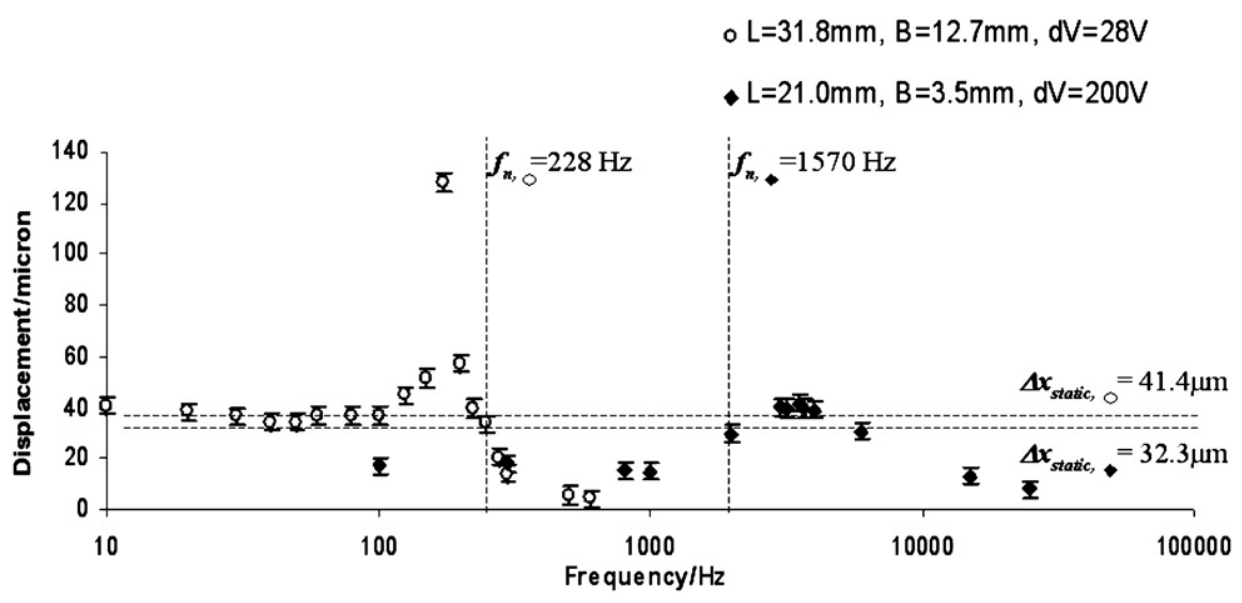

Figure 3. Influence of the excitation frequency on the amplitude of the actuator motion. The empty circles and full lozenges denote two types of boundary conditions as described in section 3.2. The actuator size and pulse amplitude are given in the legend. The dashed lines denote the theoretical values for natural frequency and static displacement.

shown in table 1 , is the surface energy of the newly created drop.

The drop formation energy is provided by the flexion work of the actuator which can be approximated as $W=0.5 F \cdot D$, where the force $F=3 d_{31} Y B T^{2} E /(8 L)$ and the maximum displacement $D=3 d_{31} L^{2} E /(8 T)$ [49] are a function of symbols described in table 1 . Also, the actuator is flexing in its first mode, with one end anchored and the other immobile along the $z$-direction so that $D$, the maximum $z$-deflection, occurs in the middle of the actuator length. For a $5 \mathrm{nl}$ drop generated from a chamber with a $20 \mathrm{~mm} \times 3.5 \mathrm{~mm}$ actuator, the efficiency $U_{d} / W$ corresponds to $0.9 \%$, which is quite low, but is a reasonable value considering the viscous dissipation in the connecting tape, the thin PDMS layer, the chamber and the main channel fluid.

\subsection{Motion of the actuator}

Given the compliant character of PDMS, we assumed in the design process that an oscillation of relatively large amplitude would be needed to produce drops. Piezoelectric bimorph actuators have large deformations by design: for instance the out-of-plane deformation (called here the $z$-displacement) is on the order of tens of micrometer for an $L=20 \mathrm{~mm}$ long actuator [49], assuming the actuator width $B \ll L$. An important parameter in the actuation design is also the eigenfrequency of the actuator, which limits the speed of deformation. The eigenfrequency $f_{n}$ of a piezoelectric bimorph with $L \gg B$ is given in [49] for two types of boundary conditions: anchored at one end (with maximum deflection at other end) as $f_{n}=\frac{0.16 T}{L^{2}} \sqrt{\frac{Y_{11}}{\rho}}$ or anchored at one end and with the other end immobile along the $z$-direction (with maximum $z$-deflection in the middle of the actuator length) as $f_{n}=\frac{0.48 T}{L^{2}} \sqrt{\frac{Y_{11}}{\rho}}$. While none of these boundary conditions corresponds exactly to the experimental conditions, where one entire side of the actuator is taped on the sealing PDMS layer of the chip (see section 2), the latter was found in better agreement with the visualized motion.
Using an Optem long distance microscope objective and a high-speed camera, we measured the temporal deformation of an actuator driven by a single rectangular pulse of amplitude $\mathrm{d} V$ and duration $(2 f)^{-1}$. Figure 3 summarizes these measurements, showing the maximum observed displacement as a function of the frequency $f$ of the driving pulse. A first series of measurements, shown by empty circles, is made for a relatively large bimorph clamped at one end, with dimensions given in figure 3 . Theoretical values are also plotted as dashed lines for both the maximum static displacement and the eigenfrequency. The agreement is relatively good in terms of resonance frequency and static (low frequency) displacement. The lower resonance frequency observed experimentally can be explained by the difficulty to perfectly anchor one end of the actuator because we used a C-clamp. A second series of measurements is made with a smaller actuator attached via double-sided tape to a $180 \mu \mathrm{m}$ thin PDMS layer, i.e. mounted as in the actual microfluidic chip. The two ends of the PDMS layer are then anchored firmly between two C-clamps, each clamp being about $1.5 \mathrm{~mm}$ away from the corresponding end of the piezo. While both the actuator size and configuration are close to the design of the microfluidic chip, the configuration is close but not exactly corresponding to the second type of boundary condition presented above: this might explain why the measured static displacement and resonance frequencies are different, both being larger than the theoretical values. Also, the recorded motion shows that the actuator ends do not move, the larger deformation occurring between these ends, confirming that the actuator vibrates in its first mode. Importantly, the visualization shows that the actuator does not freeze its motion once the driving pulse vanishes, but keeps oscillating at its natural frequency for about six periods, when the oscillations' amplitude becomes lower than the spatial measurement error. This behavior, where the chamber experiences residual oscillations, is due to the relatively large size and inertia of the actuator, and the very soft, thin PDMS sealing layer. This behavior contrasts with existing piezoelectric drop on demand dispensers and the relative modelings [50-53], where the chamber walls are 


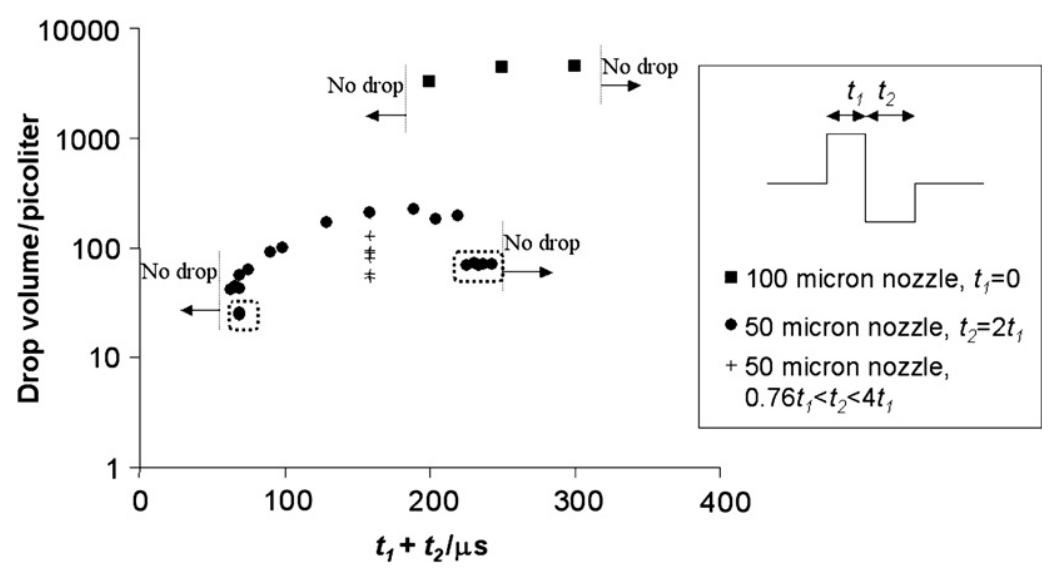

Figure 4. Drop volumes as a function of dispense parameters (nozzle size, pulse shape or length). The horizontal axis denotes the total pulse length, which involves the chamber expansion followed by the chamber compression, with respective duration $t_{1}$ and $t_{2}$. The nozzle width is indicated in the legend, with the channel height equal to the same as the nozzle width. The dotted zones show doublet dispenses, where two drops of smaller volume are generated simultaneously by a single pulse.

much stiffer, typically made of glass [50] or silicon [31]: in these cases the chamber deformation is in a direct linear relationship to the applied voltage pulse. Obviously, any theoretical modeling of the in-chip drop on demand process will have to take into account the residual oscillations of the actuator and the frequency response curve of the actuator and chamber system.

\subsection{Effects of driving pulse on the drop volumes, dispense rate and reproducibility}

In-chip drop on demand is a complex fluid dynamics process involving moving solid boundaries, acoustic wave propagation and a highly deforming liquid-liquid interface. Several aspects of the drop generation process need to be optimized in order to have a good droplet generator, such as the precise control of the drop volume and motion, the elimination of smaller satellite drops and the management of cross-talk effects in designs with multiple nozzles. Each of these aspects is sensitive to the design geometry and the actuation process [51, 53-58]. In a typical drop on demand generator, the fluidic part is made of stiff materials, which efficiently transport the pressure wave from the actuation site to the nozzle where the drop is generated. The device presented here behaves differently: the soft PDMS rubber wall reduces the apparent speed of sound in water [59] and dampens the pressure wave. This reduces cross-talk effects in chips involving multiple nozzles [60] but probably requires more energy to generate a drop (see section 3.1).

Characterization experiments reported in figure 4 describe how the drop volume is influenced by the nozzle size, the pulse shape and the pulse duration. All the data in figure 4 were obtained with an actuation voltage of $\pm 200 \mathrm{~V}$. The chamber lengths used for the respective 50 and $100 \mu \mathrm{m}$ nozzle case were $12 \mathrm{~mm}$ and $20 \mathrm{~mm}$, respectively. Note that the chamber volume is about $10 \mu \mathrm{l}$, which is about $10^{5}$ bigger than a $100 \mathrm{pl}$ volume drop, so that a pre-filled chamber can generate a large amount of drops of interest before refilling. After each dispense, we observed that the meniscus typically comes to the initial location within a few milliseconds. The shape of the pulse corresponds to an initial expansion of the chamber for a time $t_{1}$ followed by compression for a time $t_{2}$. Pulses with $t_{1}=0$ were also successful at generating a drop: they correspond to simple compression of the chamber. A quick look at the $y$-axis in figure 4 shows that drops with volumes from $25 \mathrm{pl}$ to $4.5 \mathrm{nl}$ can be generated by varying the pulse shape and the nozzle size (corresponding to the channel height): this is a remarkable range, larger than two orders of magnitude. For a given nozzle geometry, figure 4 also shows that the drop volume can be controlled smoothly by the pulse shape within one order of magnitude: for instance the $50 \mu \mathrm{m}$ nozzle produces drops in the $40-300 \mathrm{pl}$ range. Pulses with durations too different from an optimum duration will not produce any drop, as shown by the arrows in figure 4 . The reason might be that surface tension forces are strong enough to pull back the meniscus in the case of a short pulse or that a given pulse duration is needed to generate and amplify an unsteady pressure wave in the chamber $[50,51]$. For the $50 \mu \mathrm{m}$ nozzle, we observe some dual-dispense states for pulses close to the states where no drop is ejected: a dualdispense state corresponds to a case where two smaller drops are simultaneously produced, by the doublet instability process described in section 4 and shown in figure $6(d)$. Also, in the same plot, crosses demonstrate how the drop volume can be controlled by changing the ratio between the expansion time and the compression time, while keeping the total actuation time constant. We did not systematically investigate the effect of the chamber length, but we found that a long chamber $(20 \mathrm{~mm})$ produces drops in an easier manner. Experiments with a middle-length chamber $(12 \mathrm{~mm})$ produced drops for only the maximum actuation voltage, and tests with a shorter chamber $(6 \mathrm{~mm})$ did not produce any drop, although the meniscus motion was clearly visible.

The results in figures 3 and 4 suggest that the optimum pulse duration to produce drops corresponds to the natural frequency $f_{n}$ of the actuator. Indeed, the second equation presented in section 3.2 predicts values of $f_{n}$ of $1.57 \mathrm{kHz}$ and $4.74 \mathrm{kHz}$ for the actuators of the chambers with 


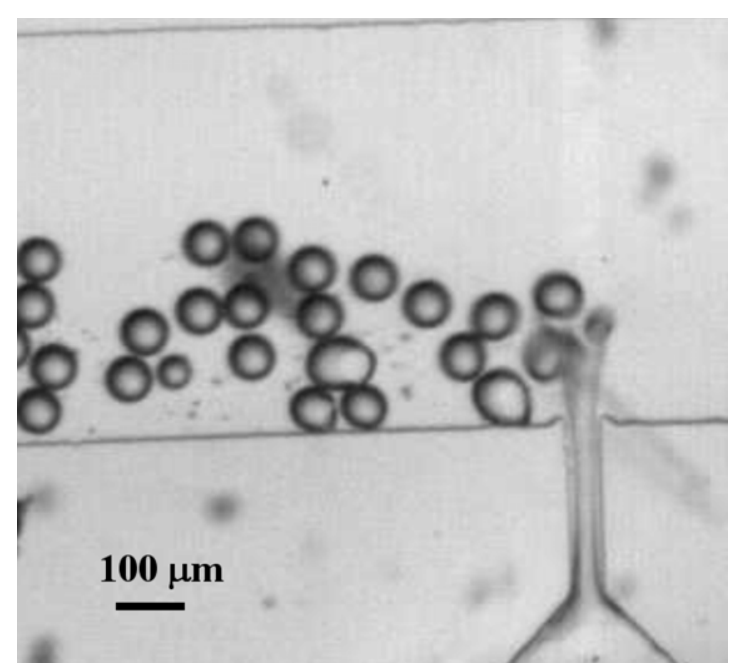

Figure 5. Dispense of individual drops by repeating the pulse at a rate of $2.5 \mathrm{kHz}$.

M An AVI movie of this figure is available from stacks.iop.org/JMM/18/065020

respectively the $100 \mu \mathrm{m}$ and $50 \mu \mathrm{m}$ nozzle. The corresponding single pulse duration for the $100 \mu \mathrm{m}$ nozzle would be $t_{2}=1 /\left(2 f_{n}\right)=318 \mu \mathrm{s}$, a time close to the 180-300 $\mu$ s interval effective at producing drops in figure 4. Similarly, the corresponding total pulse duration for the $50 \mu \mathrm{m}$ nozzle, in the case of a pulse with $t_{1}=t_{2}$, would be $t_{1}+t_{2}=1 / f_{n}=211 \mu \mathrm{s}$, a time close to the 60-220 $\mu$ s interval effective at producing drops in figure 4 . The fact that the pulse duration estimated theoretically is at the higher end of the interval of experimentally successful durations might simply indicate that the actual value of $f_{n}$ is slightly higher than the theoretical value, a fact shown in figure 3 and explained in section 3.2 by the difference of boundary conditions between the experiments and the theory.

We also tried to quantify the maximum dispense rate by repeating the driving pulse with a smaller time interval between pulses. Experiment shows that drops are still generated even if the time interval is reduced to $0 \mathrm{~s}$, which corresponds to applying the generation pulse continuously. Figure 5 and the associated movie show a case where a $400 \mu$ s pulse, shaped as in figure 4 with $t_{2}=2 t_{1}$, is applied continuously and in turn generates a train of drops at $2.5 \mathrm{kHz}$. Note that the nozzle is a $200 \mu \mathrm{m}$ long and $70 \mu \mathrm{m}$ wide straight channel. In addition, we also studied the uniformity of the drop volumes with the same nozzle. At a dispense rate of $6.2 \mathrm{~Hz}, 20$ drops generated had an average volume of $1023 \mathrm{pl}$ and a standard deviation of $16 \mathrm{pl}$, which corresponds to less than $2 \%$.

\section{Features and relevance to lab on a chip}

The process described in this paper implements in a microfluidic chip a drop on demand technique with precise, reliable control of the drop volume and generating timing. The in-chip drop on demand technique has the potential to perform in-chip reagent mixing, transport and multistep reactions. Four features of the in-chip drop on demand technique with direct relevance to lab-on-a-chip applications are presented in figure 6 and the associated movies, and are discussed in this section. The first feature in figure $6(a)$ and the associated movie is the ability to transport the dispensed drop away from the dispensing nozzle, along the main channel. This feature is realized by dispensing the drop in the main channel where the fluid is moved by a syringe pump: the measured drop velocity along the channel is $7 \mathrm{~cm} \mathrm{~s}^{-1}$. This motion of drops by viscous drag is also used in flow focusing devices [14], and is important to the in-chip drop on demand technique, because transporting a droplet from the shooting area of one nozzle to another will allow multistep reactions at frequencies of several Hertz. Interestingly, figure $6(a)$ shows that a smaller particle is embedded in the main drop, a phenomenon that can be suppressed or encouraged by adjusting the actuation pulse shape and intensity. Also, the ability of dispensing a drop that encapsulates another particle could be of interest for manufacturing complex multi-wall or hollow spheres, a topic of recent interest in microfluidics [61, 62]. Similar considerations can be made for the smaller satellite drop generated between the drop and the nozzle. Issues related to satellite formation have received abundant attention $[58,63]$ because of their relevance to printing quality.

Figure $6(b)$ and the associated movie show the second feature, which is the ability to digitally control the dispensed drop volume, by generating additional drops that coalesce with the original drop. The first frame shows a $500 \mathrm{pl}$ drop, the volume of which increases to $3.5 \mathrm{nl}$ by six successive increments of $500 \mathrm{pl}$. This coarse, digital way to control the drop volume can be coupled with the finer, analog volume control of modifying the pulse parameters (section 3.3), in order to exactly dispense the desired quantities over a wide range of volumes. While in-flight coalescence has been realized [64, 65] by atmospheric drop on demand, where drops are jetted in the air, the in-chip drop on demand technique allows a simpler realization of coalescence because the dispensed drop becomes immobile in the main channel after the kinetic energy of the dispense has been dissipated. The attentive reader probably noticed that the channel walls in figures $6(a)$ and $(b)$ are irregular: indeed these nozzles were manufactured during preliminary experiments where the master was simply a piece of electric tape applied to a glass slide and approximately cut to the desired geometry with a sharp cutter under the stereo microscope. Finally, it is worth mentioning that the large drops created in figure $6(b)$ can be split into smaller drops by moving them into a T-shaped connection [46].

The potential of mixing different reagents into a single drop is illustrated in figure $6(c)$ and the associated movie. The nozzle on the left generates a drop of ink, while a pure water drop is generated by the right nozzle simultaneously and hits the ink drop. Coalescence occurs then at $t=22 \mathrm{~ms}$, starting the mixing of the ink and water through diffusion and the transient flow associated with coalescence and the drop transport along the channel. Interestingly, coalescence does not occur right after the drops hit each other, probably 


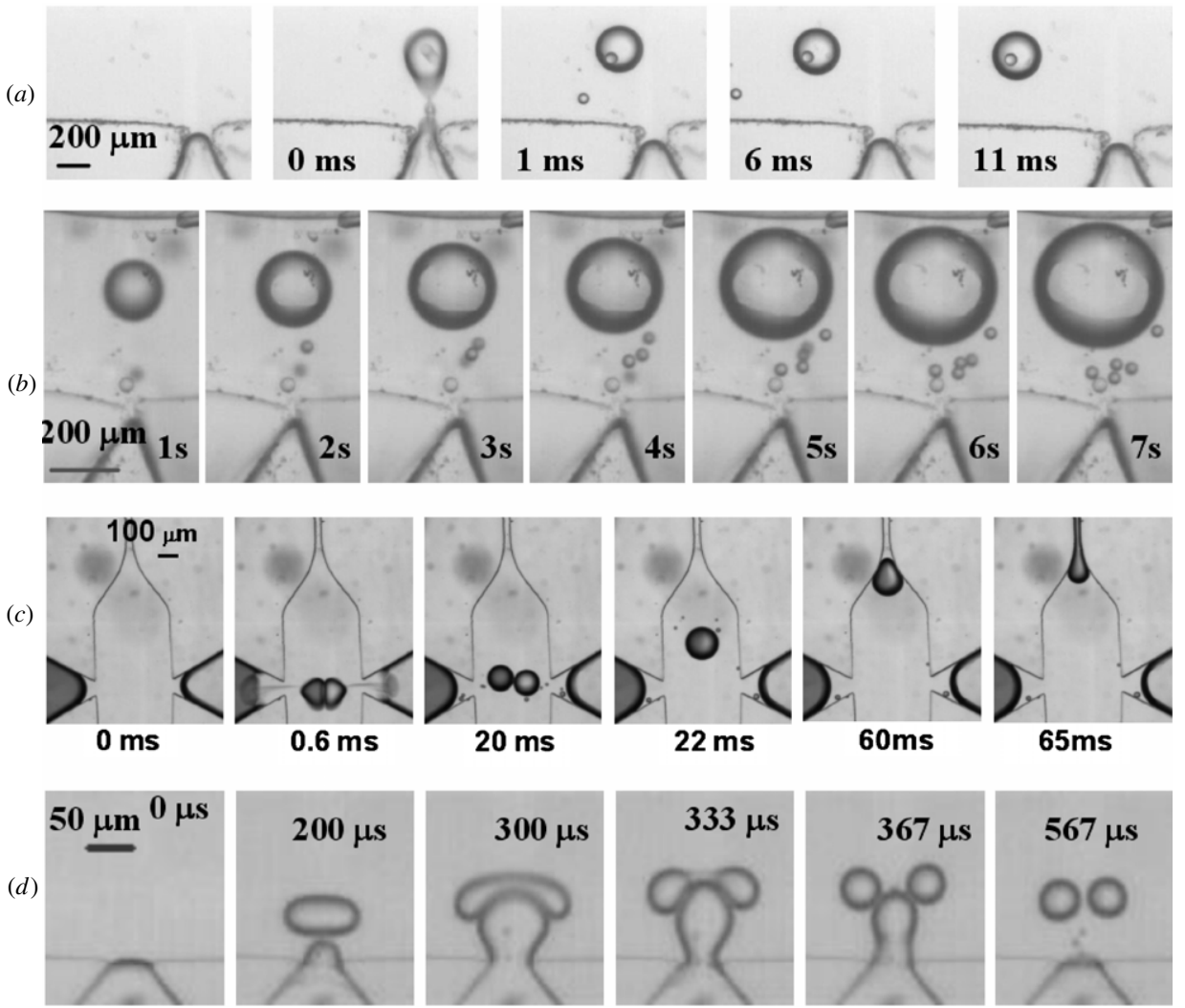

Figure 6. Four features of in-chip drop on demand with relevance to lab-on-a-chip applications. The frames are extracted from the associated movies: $(a)$ drop transport by viscous drag, $(b)$ digital control of drop volume, $(c)$ merging and mixing of two different reagents and $(d)$ doublet dispense, where two drops of small volume are generated simultaneously by a single pulse.

M] AVI movies of this figure are available from stacks.iop.org/JMM/18/065020

because of the thinning and breakup of an oil film between the two drops. This delay could be reduced by oppositely charging the two drops [23]. It is also worth mentioning that mixing and particle transport can be controlled by vibrating the liquid [66] with the same piezoelectric actuators that generate the drops. To some extent, the mixing process presented here can be compared to the impressive airborne chemistry technique, where a drop is immobilized in the air at the node of a high-power ultrasound field: this main drop acts then as an isolated reactor fed by smaller drops of reagent dispensed by atmospheric drop on demand nozzles. Airborne chemistry has been successful for screening the conditions for protein crystallization or for performing biological analyses [67-69]. Similarly, in-chip drop on demand also allows the drop dispensed in an immiscible fluid to function as an isolated reactor, fed by further reagent additions from neighboring nozzles. Major differences with the airborne chemistry technique are that optical measurements might be more difficult with the present technique, due to the presence of the hexadecane and the PDMS wall; however, the surrounding hexadecane allows higher heat transfer and suppresses evaporation.

Figure $6(d)$ and the associated movie show the fourth feature, which is the ability to generate a doublet of drops, while applying a single excitation pulse to the actuator. This occurs when an initially generated drop is hit by a strong subsequent excursion of the meniscus. During the process, the meniscus breaks the initial drop into two half drops while briefly assuming the shape of a well-known cartoon character (367 $\mu \mathrm{s})$. We call this type of dispense the doublet dispense. To the best of our knowledge, no doublet dispense has ever been realized with atmospheric drop on demand techniques because the dispensed drop quickly travels away from the nozzle area where the meniscus oscillates.

Finally, an interesting feature of the proposed in-chip drop on demand technique is that the frequencies needed to produce drops are in the order of a few $\mathrm{kHz}$ (see section 3.3), right in the audio frequency domain. Therefore, one should be able to replace the research-grade pulse generator and amplifier used to drive the actuators by inexpensive audio components: for instance, audio amplifiers are mass-produced and offer multi-channel capabilities, up to eight channels for a $\$ 300$ home cinema amplifier. We tested this hypothesis by powering the microfluidic chip with a used audio home stereo amplifier (JVC AX-R87, four channels, $400 \mathrm{~W}, \$ 37$ on a popular auction site), shown with letter ' $g$ ' in figure 2 . The pulses amplified by the audio amplifier showed minimal noise and a similar shape as the pulses amplified by the research-grade Krohn-Hite amplifier, and we managed to produce a single drop on demand (movie available from 


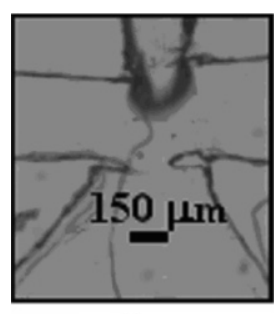

$0 \mathrm{~ms}$

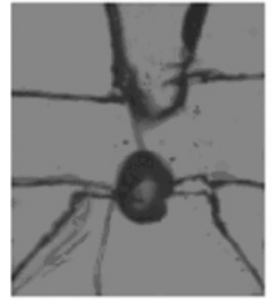

$1.4 \mathrm{~ms}$

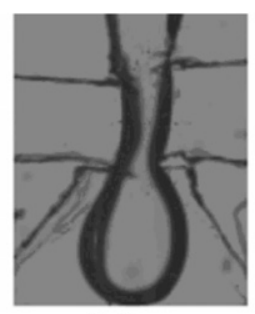

$0.8 \mathrm{~ms}$

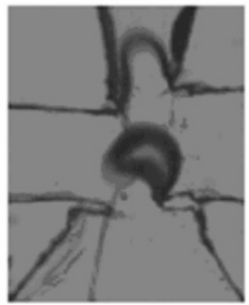

$10 \mathrm{~ms}$

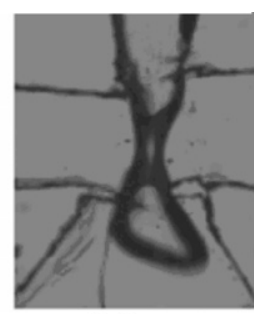

$1.2 \mathrm{~ms}$

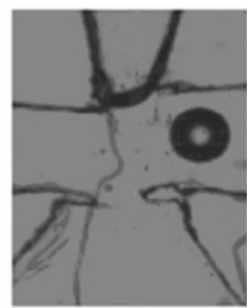

$12 \mathrm{~ms}$
Figure 7. A $1 \mathrm{nl}$ gas bubble is generated on demand in a microfluidic chip. There is no flow in the main channel.

M An AVI movie of this figure is available from stacks.iop.org/JMM/18/065020

stacks.iop.org/JMM/18/065020) by driving the actuator with the home audio amplifier connected to the function generator (d) in figure 2. This ability to drive a microfluidic chip with inexpensive, mass-produced audio electronics might seem anecdotal, however it is our conviction that the microfluidic growth will benefit from low-cost actuation schemes, which strongly contrast with the expensive components used at present.

In addition to dispensing drops, the piezoelectric actuation technique described here can be used to dispense a single bubble on demand in the main channel as shown in figure 7 and the associated movie. The bottom chamber and horizontal channel are filled with water, while the top channel is filled with air. Piezoelectric actuation of the bottom channel is used to modify the volume of the water chamber and pull the air out, which breaks up into a single bubble with the help of finger-like features next to the nozzle.

\section{Conclusion and outlook}

The in-chip drop on demand technique presented in this paper allows individual dispensing of drops of aqueous reagents or bubbles in a microfluidic chip with a temporal precision of $1 \mathrm{~ms}$ and very high rates. The ability to precisely trigger the drop generation time will allow the coordination of the generation of drops with events occurring in the chip, such as the detection of chemical reaction or temperature changes or the transit of biological cells and other particles. The drop volume can be controlled from $40 \mathrm{pl}$ to $4.5 \mathrm{nl}$ by varying the pulse shape, the chip geometry or by merging several drops together. The generated drop is surrounded by an immiscible fluid, which prevents evaporation, enhances heat transfer and can be used to transport the drop by viscous drag. Interestingly, the dead volume is quite small: a typical chamber filled with a few microliter will dispense several thousands drops with a typical volume of $100 \mathrm{pl}$.

In terms of flexibility and individuality, the proposed in-chip drop on demand technique is comparable to the digital microfluidic technique, with the advantages to work with any aqueous fluid and not only dielectric fluids, and to dispense smaller drops. In terms of mixing speed and ability to encapsulate reagents within an immiscible liquid, the in-chip drop on demand technique is comparable to segmented flow techniques, while offering more flexibility because each single droplet generation event is triggered and controlled. Finally, the ability to drive the system with inexpensive, mass-produced audio electronics is demonstrated, a feature that might help the commercial adoption of this technology.

\section{Acknowledgments}

This work has been supported partially by NSF grants 0449269 and 0701729. The last author warmly thanks his academic father, Dimos Poulikakos, who introduced him to the joy of academic research and specifically to the drop on demand technique, together with David Wallace from Microfab.

\section{References}

[1] Manz A, Harrison D J, Verpoorte E and Widmer H M 1993 Planar chips technology for miniaturization of separation systems: a developing perspective in chemical monitoring Adv. Chromatograph. 33 1-66

[2] Lion N et al 2003 Microfluidic systems in proteomics Electrophoresis 24 3533-62

[3] Paegel B M, Blazej R G and Mathies R A 2003 Microfluidic devices for DNA sequencing: sample preparation and electrophoretic analysis Curr. Opin. Biotechnol. 14 42-50

[4] Fair R 2007 Digital microfluidics: is a true lab-on-a-chip possible? Microfluid. Nanofluid. 3 245-81

[5] Song H, Chen D L and Ismagilov R F 2006 Reactions in droplets in microfluidic channels Angew. Chem. Int. Ed. Engl. 45 7336-56

[6] Verpoorte E and De Rooij N F 2003 Microfluidics meets MEMS Proc. IEEE 91 930-53

[7] Stone H A, Stroock A D and Ajdari A 2004 Engineering flows in small devices: microfluidics toward a lab-on-a-chip Ann. Rev. Fluid Mech. 36 381-411

[8] Whitesides G M 2006 The origins and the future of microfluidics Nature 442 368-73

[9] Bai X, Lee H J, Rossier J S, Reymond F, Schäfer H, Wossner M and Girault H H 2002 Pressure pinched injection of nano-liter volumes in micro-analytical devices Lab Chip 2 45-9

[10] Guenther A, Khan S, Trachsel F, Thalmann M and Jensen K 2004 Transport and reaction in microscale segmented flow Lab Chip 4 278-86

[11] Gunther A, Jhunjhunwala M, Thalmann M, Schmidt M A and Jensen K F 2005 Micromixing of miscible liquids in segmented gas-liquid flow Langmuir 21 1547-55

[12] Thorsen T, Roberts R W, Arnold F H and Quake S R 2001 Dynamic pattern formation in a vesicle-generating microfluidic device Phys. Rev. Lett. 86 4163-6

[13] Jensen M J, Stone H A and Bruus H 2006 A numerical study of two-phase Stokes flow in an axisymmetric flow-focusing device Phys. Fluids $\mathbf{1 8}$

[14] Anna S, Bontoux N and Stone H 2003 Formation of dispersions using 'flow focusing' in microchannels App. Phys. Lett. 82364 
[15] Tice J D, Song H, Lyon A D and Ismagilov R F 2003 Formation of droplets and mixing in multiphase microfluidics at low values of the Reynolds and the capillary numbers Langmuir 19 9127-33

[16] Garstecki P, Fuerstman M J, Stone H A and Whitesides G M 2006 Formation of droplets and bubbles in a microfluidic T-junction - scaling and mechanism of break-up $L a b$ Chip 6 437-46

[17] Ganan-Calvo A M 1998 Generation of steady liquid microthreads and micron-sized monodisperse sprays in gas streams Phys. Rev. Lett. 80 285-8

[18] Plateau J 1873 Statique Expérimentale et Théorique des Liquides (Paris: Gauthier-Villars)

[19] Rayleigh L 1879 Proc. Lond. Math. Soc. 10 4-13

[20] Savart F 1833 Ann. De Chim. 53 337-86

[21] Ismagilov R F, Kastrup C J, Runyon M K, Shen F and Lucchetta E 2007 Understanding complex reaction networks in space and time using microfluidics FASEB $J$. A 2142

[22] Link D R, Anna S L, Weitz D A and Stone H A 2004 Geometrically mediated breakup of drops in microfluidic devices Phys. Rev. Lett. 92054503

[23] Link D R, Grasland-Mongrain E, Duri A, Sarrazin F, Cheng Z D, Cristobal G, Marquez M and Weitz D A 2006 Electric control of droplets in microfluidic devices Angew. Chem. Int. Ed. Engl. 45 2556-60

[24] Effenhauser C, Manz A and Widmer H 1993 Glass chips for high-speed capillary electrophoresis separations with submicrometer plate heights Anal. Chem. 65 2637-42

[25] Daoura M and Meldrum D 1999 Precise automated control of fluid volumes inside glass capillaries J. Microelectromech. Syst. 8 71-7

[26] Handique K 1997 Microfluidic flow control using selective hydrophobic patterning Proc. SPIE Conf. on Micromachined Devices 3224 185-95

[27] Handique K 1998 Nanoliter-volume discrete drop injection and pumping in microfabricated analysis system Tech. Dig. of the IEEE Solid State Sensor and Actuator Workshop (Hilton Head Island, SC)

[28] Glavina P 2001 The I-STAT system: biomedical application of microsensor technology Proc. Int. MEMS Workshop 2001 iMEMS (Singapore)

[29] Puntambekar A 2001 A new fixed-volume metering microdispenser module based on sPROM's technology Proc. Transducers '01: 11th Int. Conf. Solid-State Sensors and Actuators (Munich, Germany)

[30] Aoyama R 2001 Novel liquid injection method with wedge-shaped microchannel on a PDMS microchip system for diagnostic analysis Proc. Transducers '01: 11th Int. Conf. on Solid-State Sensors and Actuators (Munich, Germany)

[31] Nguyen N and Wereley S 2002 Fundamentals of Microfluidics (Boston, MA: Artech House)

[32] He M, Edgar J S, Jeffries G D M, Lorenz R M, Shelby J P and Chiu D T 2005 Selective encapsulation of single cells and subcellular organelles into picoliter- and femtoliter-volume droplets Anal. Chem. 77 1539-44

[33] Lorenz R M, Edgar J S, Jeffries G D M and Chiu D T 2006 Microfluidic and optical systems for the on-demand generation and manipulation of single femtoliter-volume aqueous droplets Anal. Chem. 78 6433-9

[34] He M, Kuo J S and Chiu D T 2005 Electro-generation of single femtoliter- and picoliter-volume aqueous droplets in microfluidic systems App. Phys. Lett. 87031916

[35] He M, Kuo J S and Chiu D T 2006 Effects of ultrasmall orifices on the electrogeneration of femtoliter-volume aqueous droplets Langmuir 22 6408-13
[36] Gunther A and Jensen K F 2006 Multiphase microfluidics: from flow characteristics to chemical and materials synthesis Lab Chip 6 1487-503

[37] Kuhn P, Wilson K, Patch M and Stevens R 2002 The genesis of high-throughput structure-based drug discovery using protein crystallography Curr. Opin. Chem. Biol. 6 704-10

[38] Blow N 2007 Microfluidics: in search of a killer application Nature Methods 4 665-8

[39] Le H 1998 Progress and trends in ink-jet printing technology J. Imag. Sci. Technol. 42 46-92

[40] Demirci U 2006 Acoustic picoliter droplets for emerging applications in semiconductor industry and biotechnology J. Microelectromech. Syst. 15 957-66

[41] Bergkvist J, Lilliehorn T, Nilsson J, Johansson S and Laurell T 2005 Miniaturized flowthrough microdispenser with piezoceramic tripod actuation J. Microelectromech. Syst. 14 134-40

[42] Steinert C P, Goutier I, Gutmann O, Sandmaier H, Daub M, Heij B D and Zengerle R 2004 A highly parallel picoliter dispenser with an integrated, novel capillary channel structure Sensors Actuators A 116 171-7

[43] Seitz H and Heinzl J 2004 Modelling of a microfluidic device with piezoelectric actuators J. Micromech. Microeng. $141140-7$

[44] Xia Y N and Whitesides G M 1998 Soft lithography Ann. Rev. Mater. Sci. 28 153-84

[45] Carlier J, Arscott S, Thomy V, Fourrier J C, Caron F, Camart J C, Druon C and Tabourier P 2004 Integrated microfluidics based on multi-layered SU-8 for mass spectrometry analysis $J$. Micromech. Microeng. 14 619-24

[46] Garstecki P, Fuerstman M, Fischbach M A, Sia S K and Whitesides G M 2006 Mixing with bubbles: a practical technology for use with portable microfluidic devices $L a b$ Chip 6 207-12

[47] Levicky R, Friedman B, Gaspar M F A, Lee K, Patounakis G, Shepard K and Shen G 2005 Biological assaying in the near-field and with microelectronic biochips Abstracts of Papers of the American Chem. Soc. vol 230 pp U372-3

[48] Lee S, Kim D and Needham D 2001 Equilibrium and dynamic interfacial tension measurements at microscopic interfaces using a micropipet technique: 1 . A new method for determination of interfacial tension Langmuir 17 5537-43

[49] Smits J and Choi W 1991 The constituent equations of piezoelectric heterogeneous bimorphs IEEE Trans. Ultrason. Ferroelectr. Freq. Control pp 256-70

[50] Dijksman J F 1984 Hydrodynamics of small tubular pumps J. Fluid Mech. 139 173-91

[51] Bogy D B and Talke F E 1984 Experimental and theoretical study of wave propagation phenomena in drop-on-demand ink jet devices IBM J. Res. Dev. 28 314-21

[52] Hayes D J, Wallace D B and Boldman M T 1992 Picoliter solder droplet dispension Proc. ISHM Symp. '92

[53] Wallace D B 1989 A method of characteristics model of a drop-on-demand ink-jet device using an integral method drop formation model ASME Publication 89-WA/FE-4

[54] Johnson T E and Bower K W 1979 Review of the drop on-demand ink-jet with primary emphasis on the gould jet concept J. Appl. Photogr. Eng. 5 174-8

[55] Fromm J E 1984 numerical calculation of the fluid dynamics of drop-on-demand jets IBM J. Res. Dev. 28 322-33

[56] Gerhauser H, Hirschman K H, Lee F and Talke F E 1983 The effect of pulse shape on the drop volume and the frequency response of drop-on-demand ink jet transducers SID Dig. pp 110-1

[57] Chen A U and Basaran O A 2002 A new method for significantly reducing drop radius without reducing nozzle radius in drop-on demand drop production Phys. Fluids 14 L1-4 
[58] Dong H M, Carr W W and Morris J F 2006 An experimental study of drop-on-demand drop formation Phys. Fluids 18072102

[59] Wylie E B and Streeter V L 1978 Fluid Transients (New York: McGraw-Hill)

[60] Dijksman J F 1988 Hydro-acoustics of piezoelectrically driven ink-jet print heads Flow Turbul. Combust. 61 211-37

[61] Utada A, Lorenceau E, Link D, Kaplan P, Stone H and Weitz D 2005 Monodisperse double emulsions generated from a microcapillary device Science $\mathbf{3 0 8} 537$

[62] Nie Z, Xu S, Seo M, Lewis P C and Kumacheva E 2005 Polymer particles with various shapes and morphologies produced in continuous microfluidic reactors J. Am. Chem. Soc. $1278058-63$

[63] Vukasinovic B, Smith M K and Glezer A 2007 Mechanisms of free-surface breakup in vibration-induced liquid atomization Phys. Fluids 19012104

[64] Rein M 1996 The transitional regime between coalescing and splashing drops IUTAM Symp. On Waves in Liquid/Gas and Liquid/Vapor Two Phase Systems (Dordrecht: Kluwer)

[65] Morozumi Y, Nakamoto M, Fukai J and Miyatake O 2000 Numerical simulation of coalescence and separation behavior during binary droplets collision 8th Int. Conf. Liquid Atomization and Spray Systems (Pasadena, CA, USA, July 2000)

[66] Xu J and Attinger D 2007 Control and ultrasonic actuation of a gas-liquid interface in a microfluidic chip J. Micromech. Microeng. 17 609-16

[67] Santesson S and Nilsson S 2004 Airborne chemistry: acoustic levitation in chemical analysis Anal. Bioanal. Chem. 378 1704-9

[68] Santesson S, Johansson J, Taylor L S, Levander I, Fox S, Sepaniak M and Nilsson S 2003 Airborne chemistry coupled to Raman spectroscopy Anal. Chem. 75 2177-80

[69] Santesson S, Andersson M, Degerman E, Johansson T, Nilsson J and Nilsson S 2000 Airborne cell analysis Anal. Chem. 72 3412-8 